\title{
Novel cycloadducts from the 1,3-dipolar cycloaddition reactions of triazolium-1-imide 1,3-dipoles
}

\author{
Mairéad Sheridan, Paraic James,* and Cathal Healy \\ School of Chemical Sciences, Dublin City University, Dublin 9, Ireland \\ E-mail:paraic.james@dcu.ie
}

Dedicated to Tony McKervey on the occasion of his $65^{\text {th }}$ birthday

(received 28 Feb 03; accepted 22 Apr 03; published on the web 03 May 03)

\begin{abstract}
A range of imidazo-1,2,3-triazoles were synthesised by the 1,3-dipolar cycloaddition of triazolium-1-imides with $\mathrm{N}$-sulfonyl imines. Oxazolo-1,2,3-triazoles were identified as side products of this reaction. Benzyne was also used as a 1,3-dipolarophile in the cycloaddition reaction, resulting in a range of tricyclic triazoloindoles.
\end{abstract}

Keywords: 1,3-Dipolar cycloaddition, N-sulfonyl imines, benzyne

\section{Introduction}

In work previously carried out by our group 3a,6a-diaryl hexahydropyrrolotriazoles 3 underwent photoinduced disrotatory ring expansion to the new 2,5,6,7-tetrahydro-1,2,3,5 tetrazocines $\mathbf{4}^{1}$ (Scheme 1). Of key importance in the stability of these tetrazocines was the saturation of the C-6 position, and the nature of the group attached to C-6. Both saturation and electron withdrawing groups were found to initiate rearrangement of the initially formed tetrazocine, by the attack of $\mathrm{N}-2$ on C-6. In searching for new ring systems of this type, and in particular those with a fifth nitrogen in the system, the cycloadditions of triazolium-1-imides $\mathbf{1}$ with various dipolarophiles were investigated.

The cycloadditions of triazolium-1-imides with various alkene and alkyne dipolarophiles have been extensively studied. ${ }^{2}$ On addition, the initial bicyclic adducts 2 subsequently undergo a 1,4-sigmatropic rearrangement to give the bicyclic 1,2,3-triazoles 3 . 


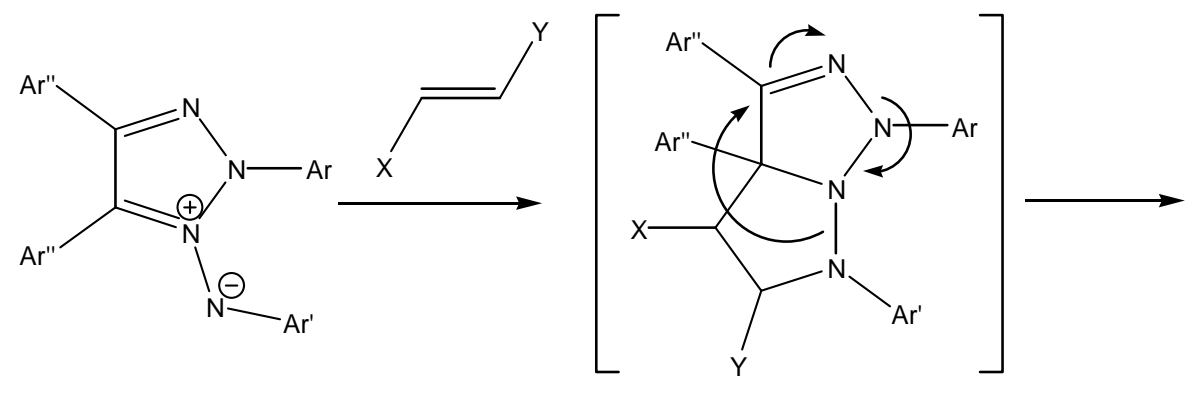

1

2
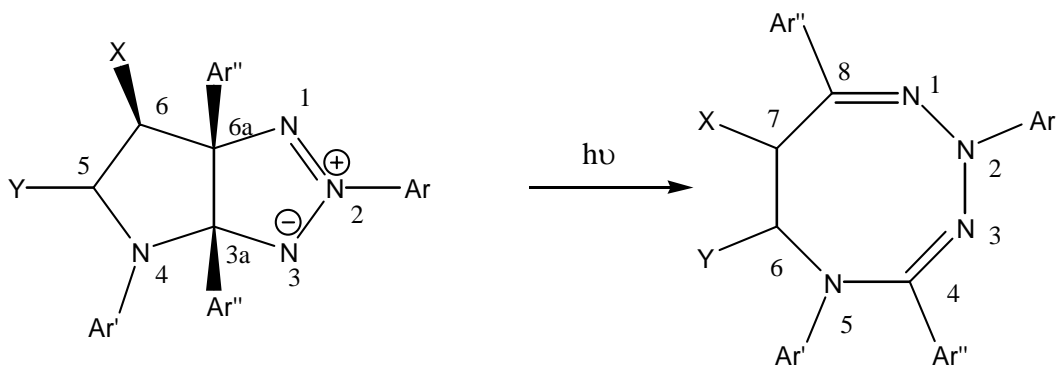

3 a $\mathrm{Ar}=\mathrm{Ar}^{\prime}=\mathrm{Ar}^{\prime \prime}=\mathrm{Ph}, \mathrm{X}=\mathrm{CO}_{2} \mathrm{Me}, \mathrm{Y}=\mathrm{H} \quad 4$

b $\mathrm{Ar}=\mathrm{Ar}^{\prime}=\mathrm{Ar} " \mathrm{Ph}, \mathrm{X}=\mathrm{CO}_{2} \mathrm{Et}, \mathrm{Y}=\mathrm{H}$

c $\mathrm{Ar}=\mathrm{Ar}^{\prime}=\mathrm{Ar}^{\prime \prime}=\mathrm{Ph}, \mathrm{X}=\mathrm{CO}_{2} \mathrm{Me}, \mathrm{Y}=\mathrm{Me}$

$\mathrm{d} \mathrm{Ar}=\mathrm{Ar}^{\prime}=\mathrm{Ar}^{\prime \prime}=\mathrm{Ph}, \mathrm{X}=\mathrm{COMe}, \mathrm{Y}=\mathrm{H}$

Scheme 1. 1,3-Dipolar cycloaddition of trizolium-1-imides, followed by 1,4-sigmatropic rearrangement. The resulting bicyclic compounds undergo photo-induced ring expansion to give the novel 1,2,3,5-tetrazocines.

\section{Results and Discussion}

\section{Benzyne as a Dipolarophile.}

The use of benzyne 5 as a dipolarophile in the cycloaddition with triazolium-1-imide $\mathbf{1}$ would provide a degree of unsaturation between C-4a and C-8a in the cycloadduct 3 (Scheme 2). Subsequent photochemical induced ring opening would produce a 1,2,3,5-tetrazocine 4 with a C5a-C-9a double bond, but attack by $\mathrm{N}-2$ on a closed aromatic sextet of electrons would be a highly unfavourable process.

In situ formation of benzyne through diazotisation of anthranilic acid $\mathbf{6}$ and isoamylnitrite in 1,2-dimethoxyethane with the 1,3-dipole 1a,b yielded the cycloadducts 3a,b. Reaction conditions were optimised by heating a stirred solution of the 1,3-dipole at the reflux temperature of the solvent, thereby increasing the reactivity of the dipole within the short transitory lifetime of benzyne. Similarly, it was observed that yields were maximised by simultaneous addition of solvent solutions of anthranilic acid and isoamylnitrite every five minutes in $2 \mathrm{~cm}^{3}$ aliquots.

Yields of the cycloadduct were increased by introducing a para nitro-group on the phenyl ring of the nitrogen terminus of the 1,3-dipole. 


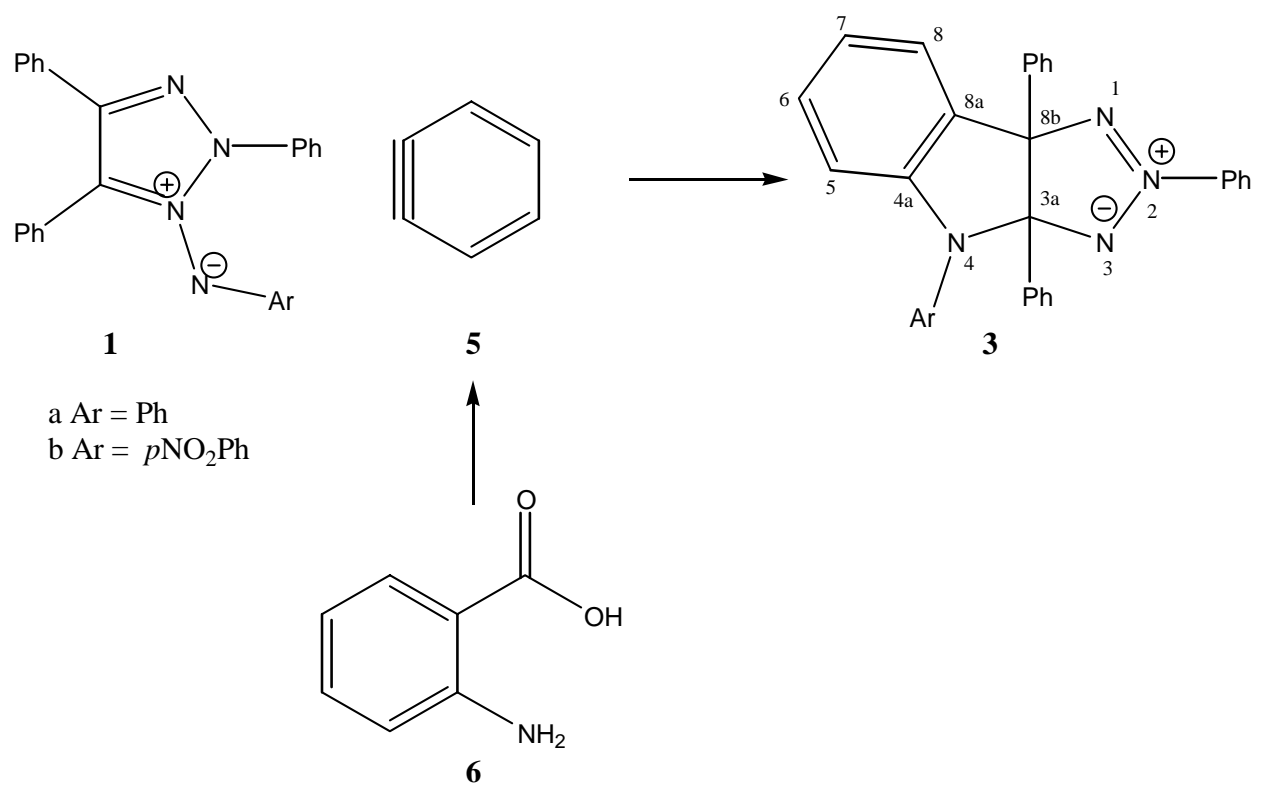

Scheme 2. 1,3-Dipolar cycloaddition of benzyne to triazolium-1-imides.

\section{N-Sulfonyl imines as dipolarophiles.}

Previously the only carbon and nitrogen-containing dipolarophiles that had been successfully used in the cycloaddition to triazolium-1-imides were isocyanates and isothiocyanates. ${ }^{2,3}$ However the use of these dipolarophiles limit the substituent at C-5 to carbonyl and thiocarbonyl groups. In order to enable variation of the substituent at C-5 a different nitrogen-containing dipolarophile was required.

$\mathrm{N}$-sulfonyl imines have been increasing in importance because they are one of the few types of electron-deficient imines that are stable enough to be isolated but reactive enough to undergo addition reactions. They have been used as electron-deficient 1,3-azabutadiene equivalents in inverse electron demand Diels-Alder chemistry, ${ }^{4}$ as electrophilic aza-aldehyde equivalents in addition reactions, ${ }^{5}$ as reactive olefin equivalents in ene reactions, ${ }^{6}$ and as precursors to Nsulfonyloxaziridines which have utility as chiral oxidants. ${ }^{7}$ It was decided to investigate if $\mathrm{N}$ sulfonyl imines could be used in the 1,3-dipolar cycloaddition with triazolium-1-imides.

Conjugation with electron-withdrawing or electron-releasing substituents increases the dipolarophilic activity of a multiple bond. Sulfonyl imines containing an aromatic group do not form adducts when heated in benzene with dienes, despite the electron-withdrawing effect of the sulfonyl group. However, if the carbon atom of the $\mathrm{C}=\mathrm{N}$ bond also carries an electronwithdrawing group as in $\mathrm{CO}_{2} \mathrm{R}, \mathrm{CF}_{3}$ or $\mathrm{CCl}_{3}$, adducts are formed under the above conditions. ${ }^{8}$ Four imines 7a-d were chosen to use as dipolarophiles in the cycloaddition reaction. Of these, three had an electron-withdrawing group 7a-c attached to the carbon, i.e. $\mathrm{CO}_{2} \mathrm{CH}_{3}, \mathrm{CO}_{2} \mathrm{C}_{2} \mathrm{H}_{5}$, $\mathrm{CCl}_{3}$, and the fourth $\mathbf{7 d}$ had a phenyl group attached to the carbon atom. As expected the $\mathrm{N}$ sulfonyl benzaldimine failed to give the required imidazo-1,2,3-triazole. The $\mathrm{N}$-sulfonyl 
trichloroimine 7c also failed to give the required adduct. However both of the N-sulfonyl imino acetic acid esters 7a,b successfully added to the triazolium-1-imide 1a-d to give the novel imidazo-1,2,3-triazoles 8a-e with a saturated C-5 position (Scheme 3). The success of the addition of the imino acetic acid esters is thought to be due to favourable secondary orbital interactions in the transition state, due to the conjugation of the $\pi$-system.

In all cases, oxazolo-1,2,3-triazoles 9a-g were isolated from the reaction mixtures. These were obtained by the hydrolysis of the N-sulfonyl imines and subsequent cycloaddition of the resulting aldehydes to the triazolium-1-imides. The use of dry solvents in inert atmosphere reduced the yield of the oxazolo-1,2,3-triazoles, but did not improve the yields of the imidazo1,2,3-triazoles. Cycloadducts of this type were previously synthesised by the cycloaddition of aldehydes with triazolium-1-imides. ${ }^{9}$ Compounds $\mathbf{9 a - e}$, and $\mathbf{9 g}$ are new derivatives of this ring system.

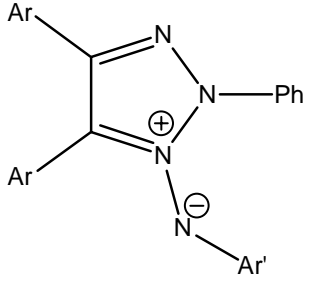

1

1a $\mathrm{Ar}=\mathrm{Ar} \mathbf{r}^{\prime}=\mathbf{P h}$

$1 \mathrm{~b} \mathrm{Ar}=\mathrm{Ph}, \mathrm{Ar}^{\prime}=p \mathrm{NO}_{2} \mathrm{Ph}$

1c $\mathrm{Ar}=\boldsymbol{p C l P h}, \mathrm{Ar}^{\prime}=\mathbf{P h}$

1d $\mathrm{Ar}=p \mathrm{CIPh}, \mathrm{Ar}^{\prime}=p \mathrm{NO}_{2} \mathrm{Ph}$

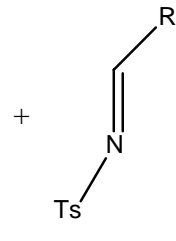

7

7a $\mathrm{R}=\mathrm{CO}_{2} \mathrm{Me}$

$7 \mathbf{b} \mathbf{R}=\mathbf{C O}_{2} \mathbf{E t}$

$7 \mathrm{c} \mathrm{R}=\mathrm{CCl}_{3}$

7d $\mathbf{R}=\mathbf{P h}$

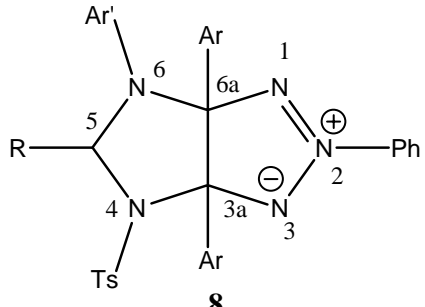

8a $\mathrm{Ar}=\mathrm{Ar} \mathbf{r}^{\prime}=\mathrm{Ph}, \mathrm{R}^{8}=\mathrm{CO}_{2} \mathrm{Me}$

$8 b \mathrm{Ar}=A r^{\prime}=\mathrm{Ph}, \mathrm{R}=\mathrm{CO}_{2} \mathrm{Et}$

8c Ar $=\mathrm{Ph}, A r^{\prime}=p \mathrm{NO}_{2} \mathrm{Ph}, \mathrm{R}=\mathrm{CO}_{2} \mathrm{Et}$

8d Ar $=p$ ClPh, Ar' $=\mathrm{Ph}, \mathrm{R}=\mathrm{CO}_{2} \mathrm{Et}$

8e Ar $=p C I P h, A r^{\prime}=p N_{2} \mathrm{Ph}, \mathrm{R}=$

$\mathrm{CO}_{2} \mathrm{Et} \quad \mathrm{Ar}^{\prime}$

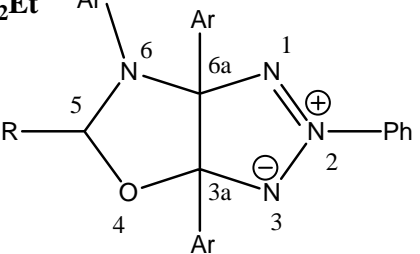

9a $\mathrm{Ar}=A \mathbf{r}^{\prime}=\mathrm{Ph}, \mathrm{R}=\mathrm{CO}_{2} \mathrm{Me}$

9b Ar $=A r^{\prime}=P h, R=C_{2} E t$

9c Ar $=$ Ph, Ar' $=p \mathrm{NO}_{2} \mathrm{Ph}, \mathrm{R}=\mathrm{CO}_{2} \mathrm{Et}$

9d Ar $=p$ ClPh, Ar' $=\mathrm{Ph}, \mathrm{R}=\mathrm{CO}_{2} \mathrm{Et}$

9e $\mathrm{Ar}=p \mathrm{CIPh}, \mathrm{Ar}^{\prime}=p \mathrm{NO}_{2} \mathrm{Ph}, \mathrm{R}=$

$\mathrm{CO}_{2} \mathrm{Et}$

9f $\mathbf{A r}=A r^{\prime}=\mathbf{R}=\mathbf{P h}$

9g Ar $=\mathrm{Ar}^{\prime}=\mathbf{P h}, \mathbf{R}=\mathrm{CCl}_{3}$

Scheme 3. 1,3-Dipolar cycladdition of triazolium-1-imides with N-sulfonyl imines, giving novel imidazo-1,2,3-triazoles. Oxazolo-1,2,3-triazoles were identified as side-products of this reaction. 
Table 1. Yields and melting points of the novel imidazo-1,2,3-triazoles and oxazolo-1,2,3triazoles

\begin{tabular}{cccccc}
\hline & $\mathrm{Ar}$ & $\mathrm{Ar}^{\prime}$ & $\mathrm{R}$ & Yield & M.P. ${ }^{\circ} \mathrm{C}$ \\
\hline $\mathbf{8 a}$ & $\mathrm{Ph}$ & $\mathrm{Ph}$ & $\mathrm{CO}_{2} \mathrm{Me}$ & $15 \%$ & $197-198$ \\
$\mathbf{8 b}$ & $\mathrm{Ph}$ & $\mathrm{Ph}$ & $\mathrm{CO}_{2} \mathrm{Et}$ & $26 \%$ & $206-208$ \\
$\mathbf{8 c}$ & $\mathrm{Ph}$ & $p \mathrm{NO}_{2} \mathrm{Ph}$ & $\mathrm{CO}_{2} \mathrm{Et}$ & $18 \%$ & $243-244$ \\
$\mathbf{8 d}$ & $p \mathrm{ClPh}$ & $\mathrm{Ph}$ & $\mathrm{CO}_{2} \mathrm{Et}$ & $10 \%$ & $228-230$ \\
$\mathbf{8 e}$ & $p \mathrm{ClPh}$ & $p \mathrm{NO}_{2} \mathrm{Ph}$ & $\mathrm{CO}_{2} \mathrm{Et}$ & $20 \%$ & $168-170$ \\
$\mathbf{9 a}$ & $\mathrm{Ph}$ & $\mathrm{Ph}$ & $\mathrm{CO}_{2} \mathrm{Me}$ & $22 \%$ & 220 \\
$\mathbf{9 b}$ & $\mathrm{Ph}$ & $\mathrm{Ph}$ & $\mathrm{CO}_{2} \mathrm{Et}$ & $25 \%$ & $154-156$ \\
$\mathbf{9 c}$ & $\mathrm{Ph}$ & $p \mathrm{NO}_{2} \mathrm{Ph}$ & $\mathrm{CO}_{2} \mathrm{Et}$ & $30 \%$ & $179-180$ \\
$\mathbf{9 d}$ & $p \mathrm{ClPh}$ & $\mathrm{Ph}$ & $\mathrm{CO}_{2} \mathrm{Et}$ & $21 \%$ & $181-182$ \\
$\mathbf{9 e}$ & $p \mathrm{ClPh}$ & $p \mathrm{NO}_{2} \mathrm{Ph}$ & $\mathrm{CO}_{2} \mathrm{Et}$ & $26 \%$ & $178-180$ \\
$\mathbf{9 f}$ & $\mathrm{Ph}$ & $\mathrm{Ph}$ & $\mathrm{Ph}$ & $50 \%$ & $171-173$ \\
$\mathbf{9 g}$ & $\mathrm{Ph}$ & $\mathrm{Ph}$ & $\mathrm{CCl}_{3}$ & $46 \%$ & $163-164$ \\
\hline
\end{tabular}

\section{Experimental Section}

General Procedures. Infrared spectra were measured on a Perkin-Elmer System 2000 FT-IR. NMR spectra were recorded on a Bruker $400 \mathrm{MHz}$ spectrometer. Melting points were recorded on a Griffin apparatus and are uncorrected. Microanalytical data was provided by the Chemistry Department in University College, Dublin. The synthesis of all dipoles ${ }^{2,10}$ and dipolarophiles ${ }^{11}$ was carried out as described previously.

The following is a typical procedure for the 1,3-dipolar cycloaddition using benzyne as the dipolarophile:

\section{2,3a,4,8b-Tetraphenyl-3,3a,4,6a-tetrahydro-[1,2,3]-triazolo[4,5-b]indole (3a)}

$1 \mathrm{~g}$ of the dipole was added to $30 \mathrm{~cm}^{3}$ of dimethoxyethane and the solution heated to reflux temperature. $5 \mathrm{~cm}^{3}$ of isoamylnitrite was added to a conical flask containing $15 \mathrm{~cm}^{3}$ of dimethoxyethane. Similarly a $20 \mathrm{~cm}^{3}$ solution of $3.5 \mathrm{~g}$ of anthranilic acid in dimethoxyethane was made up. $2 \mathrm{~cm}^{3}$ of each solution was added simultaneously to the reaction through the condenser every 5 minutes. When this was complete the reaction was further heated under vigorous stirring for 25 minutes. Excess solvent and isoamylnitrite were removed under reduced pressure and the remaining oil adsorbed onto $10 \mathrm{~g}$ of silica. The isolation of the cycloadduct was achieved by flash chromatography. ( Pet.ether (60-80) $80 \%$ :diethyl ether 20\%) to give 3a in $50 \%$ yield as a yellow solid. M.p. $142{ }^{\circ} \mathrm{C}$

$\operatorname{IR}(\mathrm{KBr})\left(\mathrm{cm}^{-1}\right)$ : 1600, 1588, 1499, 761, 774, 754. 
${ }^{1} \mathrm{H}\left(\mathrm{CDCl}_{3}\right)(\mathrm{ppm})$ : 6.81-6.93 (4H,m), 6.94-7.07 (8H,m), $7.11(1 \mathrm{H}, \mathrm{d}), 7.15-7.28(4 \mathrm{H}, \mathrm{m}), 7.44-$ 7.59(5H,m), 8.34(2H,d).

${ }^{13} \mathrm{C}\left(\mathrm{CDCl}_{3}\right)(\mathrm{ppm}): 91.40,105.42,108.92,125.42,123.31,123.97,124.05,127.51,127.58$, 127.80, 127.87, 128.34, 129.94, 129.20, 129.35, 129.64, 131.01, 132.05, 137.99, 139.24, 141.12, 141.56, 150.03 .

Microanalysis, found (theory): C 82.56 (82.73), H 4.98 (5.20), N 12.12 (12.05)

4-(4-Nitrophenyl),2,3a,8b-triphenyl-3,3a,4,6a-tetrahydro[1,2,3]-triazolo[4,5-b]indole (3b). The isolation of the cycloadduct was achieved by flash chromatograpy. ( Pet.ether (80:20) 80\%: ether $20 \%$ ) to give $3 \mathrm{~b}$ in $70 \%$ yield as a yellow solid. M.p. $158^{\circ} \mathrm{C}$ $\operatorname{IR}(\mathrm{KBr})\left(\mathrm{cm}^{-1}\right): 1585,1480,1468,1324,762,747, .685$. ${ }^{1} \mathrm{H}\left(\mathrm{CDCl}_{3}\right)(\mathrm{ppm}): 6.84-7.21(10 \mathrm{H}, \mathrm{m}), 7.27-7.36(2 \mathrm{H}, \mathrm{m}), 7.42-7.68(7 \mathrm{H}, \mathrm{m}), 8.08(2 \mathrm{H}, \mathrm{m}), 8.32(2 \mathrm{H}, \mathrm{d})$ ${ }^{13} \mathrm{C}\left(\mathrm{CDCl}_{3}\right)(\mathrm{ppm}): 120.71,122.80,123.31,125.25,127.81,127.93,128.07,128.25,128.31$, 128.38, 128.83, 129.62, 129.83, 132.21, 132.52, 136.94, 138.09, 140.78, 142.01, 147.20, 147.41. Microanalysis, found (theory): C 75.61 (75.42), H 4.33 (4.54), N 13.51 (13.74)

The following is a typical procedure for the 1,3-dipolar cycloaddition using $\mathrm{N}$-sulfonyl imines as the dipolarophile:

\section{2,3a,6,6a-Tetraphenyl-4-tosyl-5-methylcarbonyl-3,3a,4,5,6,6a-hexahydroimidazo-[4,5-d]- 1,2,3-triazole (8a)}

1,2-bis(phenylazo)stilbene (0.5g, $0.0013 \mathrm{~mol})$ and $\mathrm{N}$-p-toluenesulfonyl-2-acetic acid methyl ester $(0.35 \mathrm{~g}, 0.0015 \mathrm{~mol})$ were stirred under reflux in $25 \mathrm{~cm}^{3}$ sodium dried benzene for 24 hours. The hot solution was filtered and the filtrate evaporated to dryness. The residue was purified on a silica gel column (mobile phase, 5:1 pet. ether 40-60: ethyl acetate) yielding 123mg (0.195mmol, 15\%) of a yellow solid. M.p. $197-198^{\circ} \mathrm{C}$

${ }^{1} \mathrm{H}\left(\mathrm{CDCl}_{3}\right)(\mathrm{ppm}): 1.49\left(3 \mathrm{H}, \mathrm{s}, \mathrm{CH}_{3}\right), 3.47\left(3 \mathrm{H}, \mathrm{s}, \mathrm{OCH}_{3}\right), 5.22(1 \mathrm{H}, \mathrm{s}, \mathrm{C} 5-\mathrm{H}), 6.80-6.84(5 \mathrm{H}$, $\mathrm{m}), 6.96,(1 \mathrm{H}, \mathrm{t}, \mathrm{J}=7.2 \mathrm{~Hz}), 7.04,(2 \mathrm{H}, \mathrm{d}, \mathrm{J}=8.4 \mathrm{~Hz}), 7.12$, (2H, t, J=8.4Hz), 7.23, (3H, d, J=8.0Hz), 7.55-7.59 (6H, m), 7.65, (1H, d, J=7.2Hz), 7.98, $(2 \mathrm{H}, \mathrm{d}, \mathrm{J}=8.4 \mathrm{~Hz}), 8.45,(2 \mathrm{H}, \mathrm{d}, \mathrm{J}=8.0 \mathrm{~Hz})$ (all aromatic $\mathrm{H})$.

${ }^{13} \mathrm{C}\left(\mathrm{CDCl}_{3}\right)$ (ppm): $21.60\left(\mathrm{CH}_{3}\right), 53.00\left(\mathrm{OCH}_{3}\right), 76.70$ (C5), 97.87, 97.92 (C3a, C6a), 122.87, 124.24, 125.52, 127.30, 127.65, 127.78, 128.52, 129.03, 129.29, 129.69, 132.81, 136.02, 144.45 (all aromatic $\mathrm{C}), 164.37(\mathrm{C}=\mathrm{O})$. 
2,3a,6,6a-Tetraphenyl-4-tosyl-5-ethylcarbonyl-3,3a,4,5,6,6a-hexahydroimidazo[4,5-d]-1,2,3triazole (8b). $26 \%$ yield of a yellow solid. M.p. $206-208^{\circ} \mathrm{C}$

IR (KBr) (cm $\left.{ }^{-1}\right): 1745,1597,1505,1449,1306,1263,1168,1090,839,750,690$.

${ }^{1} \mathrm{H}\left(\mathrm{DMSO}-\mathrm{d}_{6}\right)(\mathrm{ppm}): 1.39\left(3 \mathrm{H}, \mathrm{t}, \mathrm{J}=7.2 \mathrm{~Hz}, \mathrm{OCH}_{2} \mathrm{CH}_{3}\right), 2.20\left(3 \mathrm{H}, \mathrm{s}, \mathrm{CH}_{3}\right), 4.39-4.43(2 \mathrm{H}, \mathrm{m}$, $\left.\mathrm{OCH}_{2} \mathrm{CH}_{3}\right), 5.21$ (1H, s, C5-H), $6.90(1 \mathrm{H}, \mathrm{t}, \mathrm{J}=7.2 \mathrm{~Hz}), 6.98-7.10(10 \mathrm{H}, \mathrm{m}), 7.13-7.17(4 \mathrm{H}, \mathrm{m})$, 7.45 (2H, d, J=4.8Hz), 7.61 (2H, t, 7.6Hz), 7.3 (1H, t, J=7.6Hz), 7.79 (2H, d, J=8.0Hz), 8.01 (2H, d, J=7.6Hz) (all aromatic $\mathrm{H})$.

${ }^{13} \mathrm{C}\left(\mathrm{DMSO}-\mathrm{d}_{6}\right)$ (ppm): $14.24\left(\mathrm{CH}_{3}\right), 21.31\left(\mathrm{OCH}_{2} \mathrm{CH}_{3}\right), 62.56\left(\mathrm{OCH}_{2} \mathrm{CH}_{3}\right), 75.82$ (C5), 100.35, 102.27 (С3a, С6a), 120.56, 122.64, 122.76, 127.29, 127.54, 127.79, 128.03, 128.47, 128.55, 129.14, 129.67, 129.74, 132.88, 135.77, 136.02, 138.09, 139.24, 141.13, 144.27 (all aromatic C), $171.02(\mathrm{C}=\mathrm{O})$.

Microanalysis, found, (theory): C 69.79 (69.03), H 5.30 (5.17), N 11.16 (10.88)

2,3a,6a-Triphenyl-6-(4-nitrophenyl)-4-tosyl-5-ethylcarbonyl-3,3a,4,5,6,6a

hexahydroimidazo[4,5-d]-1,2,3-triazole (8c)._18\% yield of an orange solid. M.p. $243-244^{\circ} \mathrm{C}$ IR (KBr) $\left(\mathrm{cm}^{-1}\right):$ 1739, 1597, 1472, 1450, 1540, 1310, 1175, 859.

${ }^{1} \mathrm{H}\left(\mathrm{DMSO}_{-} \mathrm{d}_{6}\right)(\mathrm{ppm}): 1.45\left(3 \mathrm{H}, \mathrm{t}, \mathrm{J}=7.2 \mathrm{~Hz}, \mathrm{OCH}_{2} \mathrm{CH}_{3}\right), 1.99\left(1 \mathrm{H}, \mathrm{s}, \mathrm{CH}_{3}\right), 4.48(2 \mathrm{H}, \mathrm{m}$ $\left.\mathrm{OCH}_{2} \mathrm{CH}_{3}\right), 5.55(1 \mathrm{H}, \mathrm{s}, \mathrm{C} 5-\mathrm{H}), 6.93-6.97$ (3H, m), 7.03-7.07 (2H, m), 7.12 (2H, t, J=4.8Hz), $7.29(2 \mathrm{H}, \mathrm{t}, \mathrm{J}=8.8 \mathrm{~Hz}), 7.61(2 \mathrm{H}, \mathrm{t}, \mathrm{J}=8.4 \mathrm{~Hz}), 7.78(2 \mathrm{H}, \mathrm{t}, \mathrm{J}=8.4 \mathrm{~Hz}), 7.85-7.94(4 \mathrm{H}, \mathrm{m}), 8.03(2 \mathrm{H}$, t, J=8.0Hz), $8.14(2 \mathrm{H}, \mathrm{d}, \mathrm{J}=9.2 \mathrm{~Hz}), 8.48(2 \mathrm{H}, \mathrm{d}, \mathrm{J}=8.0 \mathrm{~Hz}$ ) (all aromatic $\mathrm{H}$ ).

${ }^{13} \mathrm{C}\left(\mathrm{DMSO}_{6}\right)$ (ppm): $14.44\left(\mathrm{CH}_{3}\right), 21.42\left(\mathrm{OCH}_{2} \mathrm{CH}_{3}\right), 60.12\left(\mathrm{OCH}_{2} \mathrm{CH}_{3}\right), 73.15$ (C5), 96.65, 97.43 (С3a, C6a), 123.12, 124.39, 124.57, 127.40, 127.63, 127.87, 128.31, 128.41, 128.81, 129.00, 129.70, 130.23, 139.22, 144.45 (all aromatic C), $173.26(\mathrm{C}=\mathrm{O})$.

Microanalysis, found (theory): C 64.08, (64.51), H 4.51 (4.69), N 12.47 (12.20)

2,6-Diphenyl-3a,6a-di-(4-chlorophenyl)-4-tosyl-5-ethylcarbonyl-3,3a,4,5,6,6a-

hexahydroimidazo[4,5-d]-1,2,3-triazole (8d). $10 \%$ yield of an orange solid. M.p. $228-230^{\circ} \mathrm{C}$ IR (KBr) (cm $\left.{ }^{-1}\right):$ 1749, 1598, 1492, 1469, 1168, 1092, 855, 669, 768.

${ }^{1} \mathrm{H}\left(\mathrm{DMSO}_{6}\right.$ ) (ppm): 1.37 (3H, t, J=7.2Hz, $\left.\mathrm{OCH}_{2} \mathrm{CH}_{3}\right), 2.19$ (3H, s, $\left.\mathrm{CH}_{3}\right), 4.38-4.43$ (2H, m, $\left.\mathrm{OCH}_{2} \mathrm{CH}_{3}\right), 5.21(1 \mathrm{H}, \mathrm{s}, \mathrm{C} 5-\mathrm{H}), 6.93(1 \mathrm{H}, \mathrm{t}, \mathrm{J}=7.2 \mathrm{~Hz}), 7.01(2 \mathrm{H}, \mathrm{d}, \mathrm{J}=7.6 \mathrm{~Hz}), 7.07(2 \mathrm{H}, \mathrm{d}$, $\mathrm{J}=8.8 \mathrm{~Hz}), 7.12-7.20(6 \mathrm{H}, \mathrm{m}), 7.23$, $(2 \mathrm{H}, \mathrm{d}, \mathrm{J}=8.8 \mathrm{~Hz}), 7.49(2 \mathrm{H}, \mathrm{d}, \mathrm{J}=6.4 \mathrm{~Hz}), 7.60(2 \mathrm{H}, \mathrm{t}$, $\mathrm{J}=7.6 \mathrm{~Hz}), 7.74(1 \mathrm{H}, \mathrm{t}, \mathrm{J}=7.6 \mathrm{~Hz}), 7.78(2 \mathrm{H}, \mathrm{d}, \mathrm{J}=8.4 \mathrm{~Hz}), 8.05(2 \mathrm{H}, \mathrm{d}, \mathrm{J}=7.6 \mathrm{~Hz}$ ) (all aromatic H).

${ }^{13} \mathrm{C}\left(\mathrm{DMSO}_{6}\right)$ (ppm): $14.21\left(\mathrm{CH}_{3}\right), 21.31\left(\mathrm{OCH}_{2} \mathrm{CH}_{3}\right), 62.77\left(\mathrm{OCH}_{2} \mathrm{CH}_{3}\right), 75.67$ (C5), 99.70, 101.77 (С3a, С6a), 120.67, 122.83, 123.01, 127.58, 128.33, 128.54, 129.31, 129.48, 129.67, 129.84, 133.08, 133.44, 134.98, 135.56, 137.19, 139.08, 140.65, 144.49 (all aromatic C), 171.05 $(\mathrm{C}=\mathrm{O})$.

Microanalysis, found (theory): C 62.26 (62.36), H 4.47 (4.38), N 9.80 (9.83)

2-Phenyl-3a,6a-di-(4-chlorophenyl)-6-(4-nitrophenyl)-4-tosyl-5-ethylcarbonyl-3,3a,4,5,6,6ahexahydroimidazo[4,5-d]-1,2,3-triazole (8e). $20 \%$ yield of an orange solid. M.p. $168-170^{\circ} \mathrm{C}$ IR (KBr) (cm $\left.{ }^{-1}\right):$ 1763, 1598, 1470, 1509, 1323, 1173, 1115, 1092, 834, 685, 749. 
${ }^{1} \mathrm{H}\left(\mathrm{DMSO}_{\mathrm{d}}\right.$ ) (ppm): 1.45 (3H, t, J=7.2Hz, $\left.\mathrm{OCH}_{2} \mathrm{CH}_{3}\right), 2.28\left(3 \mathrm{H}, \mathrm{s}, \mathrm{CH}_{3}\right), 4.48-4.51(2 \mathrm{H}, \mathrm{m}$, $\left.\mathrm{OCH}_{2} \mathrm{CH}_{3}\right), 5.57(1 \mathrm{H}, \mathrm{s}, \mathrm{C} 5-\mathrm{H}), 6.92(2 \mathrm{H}, \mathrm{d}, \mathrm{J}=9.6 \mathrm{~Hz}), 7.05-7.09,(2 \mathrm{H}, \mathrm{m}), 7.21$ (3H, d, $\mathrm{J}=8.8 \mathrm{~Hz}), 7.30-7.35(5 \mathrm{H}, \mathrm{m}), 7.61(2 \mathrm{H}, \mathrm{d}, \mathrm{J}=8.0 \mathrm{~Hz}), 7.72(1 \mathrm{H}, \mathrm{t}, \mathrm{J}=7.2 \mathrm{~Hz}), 7.91$ (2H, d, $\mathrm{J}=8.4 \mathrm{~Hz}), 8.02-8.06(4 \mathrm{H}, \mathrm{m})($ all aromatic $\mathrm{H})$.

${ }^{13} \mathrm{C}\left(\mathrm{DMSO}-\mathrm{d}_{6}\right)(\mathrm{ppm}): 14.23\left(\mathrm{CH}_{3}\right), 21.37\left(\mathrm{OCH}_{2} \mathrm{CH}_{3}\right), 63.44\left(\mathrm{OCH}_{2} \mathrm{CH}_{3}\right), 73.21$ (C5), 100.77, 101.25 (С3a, C6a), 115.96, 123.03, 125.21, 127.64, 128.58, 129.22, 129.41, 129.55, 129.74, 129.98, 133.18, 133.29, 133.84, 134.99, 135.12, 136.87, 139.16, 139.96, 144.95, 146.13 (all aromatic $\mathrm{C}), 170.78(\mathrm{C}=\mathrm{O})$.

The following is a typical procedure for the 1,3-dipolar cycloaddition with aldehydes acting as the dipolarophile.

\section{2,3a,5,6,6a-Pentaphenyl-3,3a,5,6-tetrahydro-oxazolo-[5,4-d]-1,2,3-triazole (9f)}

1,2-bis(phenylazo)stilbene $(0.4 \mathrm{~g}, 0.001 \mathrm{~mol})$ and $\mathrm{N}$-p-toluenesulfonyl benzaldimine $(0.26 \mathrm{~g}$, $0.001 \mathrm{~mol}$ ) were stirred under reflux in sodium-dried benzene for 6 hours. After 6 hours a further 0.026g (0.0001mol, 10\% excess) of $\mathrm{N}$-p-toluenesulfonyl benzaldimine was added and the mixture allowed to reflux for a further 12 hours. The benzene was removed under vacuum and the residue was recrystallised from ethanol, yielding $0.24 \mathrm{~g}(0.0005 \mathrm{~mol}, 50 \%)$ of a yellow solid. M.p. $171-173^{\circ} \mathrm{C}$ (lit. $\left.174-175^{\circ} \mathrm{C}\right)^{9}$

IR (KBr) $\left(\mathrm{cm}^{-1}\right): 1602,1501,1450,763,691$.

${ }^{1} \mathrm{H}$ (DMSO-d (D) (pp): 6.28 (1H, s, C5-H), 6.78 (3H, t, J=4Hz), 6.99 (2H, t, J=8Hz), 7.14 (6H, t, $\mathrm{J}=8 \mathrm{~Hz}), 7.21(2 \mathrm{H}, \mathrm{t}, \mathrm{J}=8 \mathrm{~Hz}), 7.47-7.57(5 \mathrm{H}, \mathrm{m}), 7.68(2 \mathrm{H}, \mathrm{t}, \mathrm{J}=8 \mathrm{~Hz}), 7.76(1 \mathrm{H}, \mathrm{t}, \mathrm{J}=8 \mathrm{~Hz}), 7.93$ (2H, d, J=8Hz), 8.25 (2H, d, J=8Hz), (all aromatic $\mathrm{H}$ ).

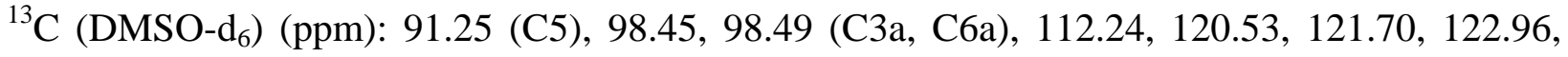
126.95, 127.56, 127.77, 128.29, 129.44, 129.28, 129.86, 129.97, 132.89, 137.19, 138.28, 138.89, 140.33, 143.33 (all aromatic C).

\section{2,3a,6,6a-Tetraphenyl-5-methylcarbonyl-3,3a,5,6-tetrahydro-oxazolo-[5,4-d]-1,2,3-triazole}

(9a). $22 \%$ yield of a pale yellow solid. M.p. $220^{\circ} \mathrm{C}$

IR (KBr) $\left(\mathrm{cm}^{-1}\right):$ 1753, 1598, 1505, 1449 , 752, 698.

${ }^{1} \mathrm{H}\left(\mathrm{DMSO}_{6} \mathrm{~d}_{6}\right)(\mathrm{ppm}): 3.97$ (3H, s, OCH $), 5.69$ (1H, s, C5-H), $6.61(2 \mathrm{H}, \mathrm{d}, \mathrm{J}=8.4 \mathrm{~Hz}), 6.77(1 \mathrm{H}$, t, J=7.2Hz), 7.05-7.15 (10H, m), $7.45(2 \mathrm{H}, \mathrm{d}, \mathrm{J}=6.4 \mathrm{~Hz}), 7.64(2 \mathrm{H}, \mathrm{t}, \mathrm{J}=7.2 \mathrm{~Hz}), 7.73(1 \mathrm{H}, \mathrm{t}$, $\mathrm{J}=7.2 \mathrm{~Hz}), 8.22(2 \mathrm{H}, \mathrm{d}, \mathrm{J}=7.6 \mathrm{~Hz})$ (all aromatic $\mathrm{H})$.

${ }^{13} \mathrm{C}$ (DMSO-d $)$ (ppm): $53.73\left(\mathrm{OCH}_{3}\right), 86.76$ (C5), 97.08, 114.78 (C3a, C6a), 115.76, 119.94, 123.01, 126.89, 127.23, 127.85, 128.35, 128.48, 128.79, 129.07, 129.89, 133.04, 136.02, 137.26, 140.22, 141.34 (all aromatic C), $170.40(\mathrm{C}=\mathrm{O})$.

Microanalysis, found (theory): C 72.79 (73.09), H 5.17 (5.08), N 11.65 (11.76) 


\section{2,3a,6,6a-Tetraphenyl-5-ethylcarbonyl-3,3a,5,6-tetrahydro-oxazolo-[5,4-d]-1,2,3-triazole}

(9b). $25 \%$ yield of a yellow solid. M.p. $154-156^{\circ} \mathrm{C}$

IR (KBr) $\left(\mathrm{cm}^{-1}\right):$ 1754, 1600, 1505, 1449, 1232, 1203, 1180, 753, 699.

${ }^{1} \mathrm{H}\left(\mathrm{DMSO}-\mathrm{d}_{6}\right)(\mathrm{ppm}): 1.37\left(3 \mathrm{H}, \mathrm{t}, \mathrm{J}=7.2 \mathrm{~Hz}, \mathrm{OCH}_{2} \mathrm{CH}_{3}\right), 4.41-4.46\left(2 \mathrm{H}, \mathrm{m} \mathrm{OCH} \mathrm{CH}_{3}\right), 5.65$ $(1 \mathrm{H}, \mathrm{s}, \mathrm{C}), 6.29(2 \mathrm{H}, \mathrm{d}, \mathrm{J}=8.0 \mathrm{~Hz}), 6.77(1 \mathrm{H}, \mathrm{t}, \mathrm{J}=7.6 \mathrm{~Hz}), 7.08-7.13(10 \mathrm{H}, \mathrm{m}), 7.46(2 \mathrm{H}, \mathrm{d}$, $\mathrm{J}=5.2 \mathrm{~Hz}), 7.64(2 \mathrm{H}, \mathrm{t}, \mathrm{J}=8.0 \mathrm{~Hz}), 7.73(1 \mathrm{H}, \mathrm{d}, \mathrm{J}=7.2 \mathrm{~Hz}), 8.22(2 \mathrm{H}, \mathrm{d}, \mathrm{J}=7.2 \mathrm{~Hz})$ (all aromatic H).

${ }^{13} \mathrm{C}\left(\mathrm{DMSO}-\mathrm{d}_{6}\right)(\mathrm{ppm}): 14.32\left(\mathrm{OCH}_{2} \mathrm{CH}_{3}\right), 62.65\left(\mathrm{OCH}_{2} \mathrm{CH}_{3}\right), 86.96$ (C5), 97.11, $114.79(\mathrm{C} 3 \mathrm{a}$, C6a), 115.73, 119.91, 123.02, 126.91, 127.25, 127.82, 128.31, 128.47, 128.78, 129.05, 129.89, 133.02, 136.09, 137.25, 140.23, 141.33 (all aromatic C), $169.86(\mathrm{C}=\mathrm{O})$.

Microanalysis, found (theory): C 73.08 (73.45), H 5.69 (5.34), N 10.74 (11.42)

2,3a,6a-Triphenyl-6-(4-nitrophenyl)--5-ethylcarbonyl-3,3a,5,6-tetrahydro-oxazolo-[5,4-d]1,2,3-triazole (9c). $30 \%$ yield of a yellow solid. M.p. $179-180^{\circ} \mathrm{C}$ IR (KBr) (cm $\left.{ }^{-1}\right)$ : 1754, 1597, 1449, 1505, 1385, 1314, 1211, 1160, 1134, 1114, 834, 758, 702.

${ }^{1} \mathrm{H}\left(\mathrm{DMSO}-\mathrm{d}_{6}\right)(\mathrm{ppm}): 1.41$ (3H, t, J=7.2Hz, OCH $\left.\mathrm{CH}_{3}\right), 4.48\left(2 \mathrm{H}, \mathrm{m}, \mathrm{OCH}_{2} \mathrm{CH}_{3}\right), 5.95(1 \mathrm{H}, \mathrm{s}$, C5), 6.73 (2H, d, J=9.2Hz), 7.03-7.15 (9H, m), $7.65(2 \mathrm{H}, \mathrm{t}, \mathrm{J}=8.0 \mathrm{~Hz}), 7.73(1 \mathrm{H}, \mathrm{d}, \mathrm{J}=6.8 \mathrm{~Hz})$, 8.05 (2H, d, 9.2Hz), 8.25 (2H, d, J=8.0Hz) (all aromatic $\mathrm{H})$.

${ }^{13} \mathrm{C}\left(\mathrm{DMSO}-\mathrm{d}_{6}\right)(\mathrm{ppm}): 18.90\left(\mathrm{OCH}_{2} \mathrm{CH}_{3}\right), 63.15\left(\mathrm{OCH}_{2} \mathrm{CH}_{3}\right), 86.34$ (C5), 96.91, 114.99 (C3a, C6a), 115.20, 123.13, 125.41, 126.77, 127.12, 128.01, 128.58, 128.93, 129.91, 133.23, 135.29, 135.84, 139.51, 140.10, 146.80 (all aromatic C), $168.90(\mathrm{C}=\mathrm{O})$.

Microanalysis, found (theory): C 67.00 (67.28), H 4.79 (4.71), N 12.87 (13.08)

2,6-Diphenyl-3a,6a-di-(4-chlorophenyl)-5-ethylcarbonyl-3,3a,5,6-tetrahydro-oxazolo-[5,4d]-1,2,3-triazole (9d). $21 \%$ yield of a yellow solid. M.p. $181-182^{\circ} \mathrm{C}$ IR (KBr) $\left(\mathrm{cm}^{-1}\right)$ : 1741, 1598, 1492, 1468, 1305, 1236, 1092, 832, 746, 685.

${ }^{1} \mathrm{H}\left(\mathrm{DMSO}-\mathrm{d}_{6}\right)(\mathrm{ppm}): 1.35\left(3 \mathrm{H}, \mathrm{t}, \mathrm{J}=6.8 \mathrm{~Hz}, \mathrm{OCH}_{2} \mathrm{CH}_{3}\right), 4.37-4.48\left(2 \mathrm{H}, \mathrm{m}, \mathrm{OCH}_{2} \mathrm{CH}_{3}\right), 5.68$ (1H, s, C5), 6.61 (2H, d, J=8.8Hz), $6.80(1 \mathrm{H}, \mathrm{t}, \mathrm{J}=7.2 \mathrm{~Hz}), 7.11-7.16(4 \mathrm{H}, \mathrm{m}), 7.23-7.26(4 \mathrm{H}, \mathrm{m})$, $7.48(2 \mathrm{H}, \mathrm{d}, \mathrm{J}=7.6 \mathrm{~Hz}), 7.64(2 \mathrm{H}, \mathrm{t}, \mathrm{J}=8.0 \mathrm{~Hz}), 7.73(1 \mathrm{H}, \mathrm{t}, \mathrm{J}=6.8 \mathrm{~Hz}), 8.22(2 \mathrm{H}, \mathrm{d}, \mathrm{J}=7.6 \mathrm{~Hz})$ (all aromatic $\mathrm{H})$.

${ }^{13} \mathrm{C}\left(\mathrm{DMSO}-\mathrm{d}_{6}\right)(\mathrm{ppm}): 14.29\left(\mathrm{OCH}_{2} \mathrm{CH}_{3}\right), 62.81\left(\mathrm{OCH}_{2} \mathrm{CH}_{3}\right), 86.97$ (C5), 96.73, 114.35 (C3a, C6a), 115.89, 120.28, 123.11, 128.12, 128.58, 128.88, 129.20, 129.87, 133.16, 133.28, 133.71, 135.09, 136.29, 140.12, 140.94 (all aromatic C), $169.83(\mathrm{C}=\mathrm{O})$.

Microanalysis, found (theory): C 64.35 (64.41), H 4.44 (4.32), N 9.76 (10.01)

2-Phenyl-3a,6a-di-(4-chlorophenyl)-6-(4-nitrophenyl)-5-ethylcarbonyl-3,3a,5,6-tetrahydrooxazolo-[5,4-d]-1,2,3-triazole (9e). 26\% yield of a yellow solid. M.p. $178-180^{\circ} \mathrm{C}$

IR (KBr) $\left(\mathrm{cm}^{-1}\right):$ 1747, 1600, 1466, 1509, 1326, 1173, 1137, 1115, 1094, 752, 690.

${ }^{1} \mathrm{H}\left(\mathrm{DMSO}-\mathrm{d}_{6}\right)(\mathrm{ppm}): 1.39\left(3 \mathrm{H}, \mathrm{t}, \mathrm{J}=6.8 \mathrm{~Hz}, \mathrm{OCH}_{2} \mathrm{CH}_{3}\right), 4.46-4.50\left(2 \mathrm{H}, \mathrm{m}, \mathrm{OCH}_{2} \mathrm{CH}_{3}\right), 5.98$ (1H, s, C5), $6.71(2 \mathrm{H}, \mathrm{d}, \mathrm{J}=9.2 \mathrm{~Hz}), 7.08-7.15(4 \mathrm{H}, \mathrm{m}), 7.25-7.30(4 \mathrm{H}, \mathrm{m}), 7.65$ (2H, t, J=8.0Hz), $7.74(1 \mathrm{H}, \mathrm{t}, \mathrm{J}=7.2 \mathrm{~Hz}), 8.09$ (2H, d, J=9.6Hz), 8.24 (2H, d, J=7.6Hz) (all aromatic H). 
${ }^{13} \mathrm{C}\left(\mathrm{DMSO}-\mathrm{d}_{6}\right)(\mathrm{ppm}): 14.29\left(\mathrm{OCH}_{2} \mathrm{CH}_{3}\right), 62.79\left(\mathrm{OCH}_{2} \mathrm{CH}_{3}\right), 86.97$ (C5), 96.74, 114.36 (C3a, C6a), 115.89, 120.25, 123.12, 128.11, 128.58, 128.89, 129.19, 129.85, 133.14, 133.27, 133.70, 135.11, 136.30, 140.13, 140.95 (all aromatic C), $169.83(\mathrm{C}=\mathrm{O})$.

Microanalysis, found (theory): C 59.72 (59.60), H 4.05 (3.84), N 11.12 (11.59)

\section{2,3a,6,6a-Tetraphenyl-5-trichloromethyl-3,3a,5,6-tetrahydro-oxazolo-[5,4-d]-1,2,3-triazole}

(9g). 46\% yield of an off-white solid. M.p. $163-164^{\circ} \mathrm{C}$

IR (KBr) $\left(\mathrm{cm}^{-1}\right):$ 1589, 1487, 1461, 767, 697.

${ }^{1} \mathrm{H}$ (DMSO-d (D) (ppm): 6.28 (1H, s, C5-H), 7.05-7.28 (13H, m), 7.50 (2H, d, J=8Hz), 7.68 (2H, d, $\mathrm{J}=8 \mathrm{~Hz}), 7.76(1 \mathrm{H}, \mathrm{t}, \mathrm{J}=8 \mathrm{~Hz}), 8.24(2 \mathrm{H}, \mathrm{d}, \mathrm{J}=8 \mathrm{~Hz})$, (all aromatic $\mathrm{H})$.

${ }^{13} \mathrm{C}$ (DMSO-d $)$ (ppm): 97.15 (C5), 102.04, 102.30 (C3a, C6a), 112.50, 124.09, 127.87, 128.63, 128.77, 129.32, 129.55, 130.11, 130.74, 133.86, 137.87, 138.74, 141.35, 144.28 (all aromatic C). Microanalysis found (theory):C 62.58 (62.76), H 3.93 (3.95), N 10.34 (10.46)

\section{Acknowledgments}

M. Sheridan and C. Healy acknowledge Irish government funding.

\section{References}

1. Byrne, C.; Draper, S.M.; James, J.P.; Long, C. J. Chem. Res. 1995, 2501.

2. (a) Angadiyavar, C.S.; Sukumaran, K.B.; George, M.V. Tetrahedron Lett. 1971, 633. (b) Sukumaran, K.B.; Angadiyavar, C.S.; George, M.V. Tetrahedron 1972, 28, 3987. (c) Butler, R.N.; Cunningham, D.; James, J.P.; McArdle, P. J. Chem. Soc., Chem. Commun. 1983, 762.

3. (a) Butler, R.N.; Colleran, D.M. J. Chem. Soc., Perkin Trans. 1 1992, 2159. (b) Butler, R.N.; Grogan, D.C.; McDonald, P.D.; Burke, L.A. J. Chem. Soc., Perkin Trans. 1 1997, 3587.

4. (a) Albrecht, R.; Kresze, G. Chem. Ber. 1964, 12, 949. (b) Boger, D.L.; Weinreb, S.N. Hetero Diels-Alder Methodology in Organic Synthesis, Academic: San Diego, 1987. (c) Boger, D.L.; Kasper, A.m. J. Am. Chem. Soc. 1989, 111, 1517. (d) Boger, D.L.; Corbett, W.L.; Curran, T.T.; Kasper, A.M. J. Am. Chem. Soc. 1991, 113, 1713.

5. Sisko, J.; Weinreb, S.M. J. Org. Chem. 1990, 55, 393.

6. (a) Tschaen, D.M.; Turos, E.; Weinreb, S.M. J. Org. Chem. 1984, 49, 5058. (b) Melnick, M.J.; Freyer, A.J.; Weinreb, S.M. Tetrahedron Lett. 1988, 29, 3891.

7. Davis, F.A.; Reddy, R.T.; Reddy, R.E. J. Org. Chem. 1992, 57, 6387.

8. Kresze, G.; Wucherpfennig, W. Angew. Chem., Int. Ed. 1967, 6, 149.

9. Butler, R.N.; O'Shea, D.F. J. Chem. Soc. Perkin Trans 1 1994, 2797.

10. (a) Sukumaran, K.B.; Satish, S.; George, M.V. Tetrahedron 1974, 30, 445. (b) Alexandrou, N.E. Tetrahedron 1966, 22, 1309. 
11. (a) Albrecht, R.; Kresze, G.; Mlakar, B. Chem. Ber. 1965, 98, 1431. (b) Baillargé, M.; Le Goffic, F. Synth. Commun. 1987, 17, 1603. (c) Love, B.E.; Raje, P.S.; Williams, T.C. Synlett 1994, 493. 